\title{
Exposure to green space and prevention of anxiety and depression among young people in urban settings: a global scoping review
}

\author{
Rebecca Reece, Isabelle Bray, Danielle Sinnett, Robert Hayward and Faith Martin
}

Rebecca Reece, Isabelle Bray, Danielle Sinnett and Robert Hayward all are based at the University of the West of England, Bristol, UK. Faith Martin is based at Coventry University, Coventry, UK.

Received 26 February 2021 Revised 11 March 2021 Accepted 15 March 2021

() Rebecca Reece, Isabelle Bray, Danielle Sinnett, Robert Hayward and Faith Martin. Published by Emerald Publishing Limited. This article is published under the Creative Commons Attribution (CC BY 4.0) licence. Anyone may reproduce, distribute, translate and create derivative works of this article (for both commercial and non-commercial purposes), subject to full attribution to the original publication and authors. The full terms of this licence may be seen at http://creativecommons. org/licences/by/4.0/legalcode

This work was supported by a Wellcome Trust Mental Health Priority Area "Active Ingredients" commission awarded to IB at the University of the West of England, Bristol. The authors would like to thank Pauline Shaw, Subject Librarian at UWE, Bristol, for her advice with the literature searches for the scoping review and

literature review.

\begin{abstract}
Purpose - There is a mental health crisis, particularly among young people. Despite many young people living in urban settings, reviews about the association between exposure to green or natural environments and mental health tend to focus on either children or adults. The aim of this review is to examine the scope of the global literature for this age group, to inform a systematic review on the role of exposure to green space in preventing anxiety and depression amongst young people aged 14-24 years.

Design/methodology/approach - Seven databases were searched for quantitative and qualitative sources published from January 2000 to June 2020. This identified 201 sources and their characteristics are described here. Gaps in the literature are also highlighted.

Findings - The number of relevant studies published per year has increased over time. Most studies are set in North America (28\%) or Europe (39\%). The most common study designs were observational (34\%) or experimental (28\%). A wide range of exposures and interventions are described.

Research limitations/implications - This review included literature from predominantly high-income countries and has shown the under-representation of low-middle income countries and lack of ethnic diversity in study populations. It has also highlighted the lack of clinical measures of anxiety and depression as outcomes.

Originality/value - This inter-disciplinary review has contributed to the field by describing the geographic distribution of the literature and the broad range of exposures to green spaces being reported. Unlike previous scoping reviews, this review focused specifically on young people and on measures of anxiety and depression and their pre-cursers.
\end{abstract}

Keywords Mental health, Young people, Global scoping review, Green spaces, Natural environments Paper type Literature review

\section{Introduction}

Living in an urban environment is a risk factor for poorer mental health (Ventriglio et al., 2021; Lecic-Tosevski, 2019; Krefis et al., 2018). There is a 20\% higher risk of developing depression in individuals who live in urbanised areas compared to rural areas (Sundquist et al., 2018). Today, $55 \%$ of the world's population lives in urban areas, a proportion that is expected to increase to 68\% by 2050 (UN Department of Economic and Social Affairs, 2018). Given that $16 \%$ of the 7.8 billion world population is aged 14-24 years (Worldometer, 2020), and that young people are more likely to live in urban areas (Thomas et al., 2015), there are at least 0.7 billion 14-24-year olds living in urban settings globally. Promoting good mental health in young people is important as $20 \%$ of children and adolescents globally live with a mental health condition (WHO, 2020). In 2017, 31\% of young women 16-24 years in the UK reported evidence of anxiety or depression [Office for National Statistics (ONS), 2020], which are 
comorbid conditions (Tiller, 2013). In addition, amongst 15-29-year-olds, suicide is the second leading cause of death (World Health Organisation, WHO, 2020). It is particularly important to improve the mental health of young people, as many adults with mental health problems develop these during adolescence (Kessler et al., 2007; Patel et al, 2007). A preventative approach to mental health in young people is important because it could save lives and be a cost-effective solution to reducing the burden that mental health problems have on society (Jacka and Reavley, 2014).

Exposure to urban green space and mental health in young people is especially important during the current Covid-19 pandemic. This has influenced the level of experienced anxiety in university students (Cao et al., 2020). It has further highlighted the need for access to parks and green spaces during the Covid-19 pandemic and the importance of these spaces for mental health benefits (Slater et al., 2020). Additionally, the densification of cities reduces availability of private and public green spaces and this increases mental health problems associated with urban living (Srivastava, 2009). It is vital to better understand this relationship, and the Covid-19 pandemic has increased this urgency.

Several systematic reviews provide evidence that exposure to green space and connection with nature can benefit mental well-being, but they focus on either children (McCormick, 2017 (0-18years); Tillmann et al., 2018 (0-18years); ) or adults (Corazon et al., 2019 (18-86 years); Thompson Coon et al., 2011 ( $\geq 18$ years)), and it is not clear to what extent the results from these age groups can be generalised to the 14-24 age range. Other systematic reviews include children and young people (Vanaken and Danckaerts, 2018) (3-25years) or young people and adults (Houlden et al., 2018) (12-99years). Although these reviews overlap with the 14-24 age range, no previous reviews focus on young people of this age specifically.

Systematic reviews focusing on adult populations have shown green spaces and outdoor nature-based interventions can have positive associations with mental well-being and emotional measures related to stress relief (Corazon et al., 2019; Houlden et al., 2018), as well as some positive benefits to mental well-being when exercising outdoors compared to indoors (Thompson Coon et al., 2011). Regarding children and adolescents, previous systematic reviews have shown beneficial associations between exposure and access to green space and mental health outcomes, mental well-being, cognitive development and emotional and behavioural difficulties (Tillmann et al., 2018; McCormick, 2017; Vanaken and Danckaerts, 2018). However, there is less research and a lack of reviews focusing on the role of exposure to green space in preventing anxiety and depression in young people living in urban settings. Further, the range of exposures and interventions included in previous reviews tends to be limited (e.g. single episodes of walking/running indoors and outdoors, measuring proximity of green spaces with land cover maps, views of green spaces from home, reported frequency of visiting green spaces). A systematic review for young people aged 14-24 living in urban settings, considering a broader range of exposures to green and natural environments and including activities undertaken in these environments, would fill a gap in the literature and inform built environment policymakers and practitioners as well as healthcare professionals. As the literature is multidisciplinary, complex and heterogenous, a scoping review is needed to describe the existing evidence base and to provide the first step towards conducting a systematic review (Mays et al., 2001; Arksey and O'Malley, 2005).

Two recent scoping reviews consider the mental health benefits of green spaces (Wendelboe-Nelson et al., 2019; Callaghan et al., 2020). Both reviews found that the majority of studies showed a positive association between mental health outcomes and exposure to green space. However, the first included adults (Wendelboe-Nelson et al., 2019 $(\geq 18$ years)). The second scoping review focused on urban settings in Europe but had a primary care focus and included studies of adults as well as young people (Callaghan et al., 2020 ( $\geq 15$ years)). Therefore, this scoping review will focus on young people aged 
14-24years. In terms of outcomes, it will focus on two common mental health disorders, anxiety and depression (World Health Organisation, WHO, 2017), as well as precursors of these conditions such as "low mood".

Evidence from different disciplines, countries, and study designs (quantitative and qualitative) will be considered to answer the research question:

$R Q 1$. What is the state of the evidence base for exposure to green space to reduce the risk of anxiety and depression among young people living in urban settings?

\section{Methods}

This scoping review followed the guidance and items presented in the "Preferred Reporting Items for Systematic Reviews and Meta-Analyses Extension for Scoping Reviews (PRISMA-ScR)" checklist (Tricco et al., 2018).

\subsection{Searches}

The following databases were searched to identify relevant peer-reviewed sources: Medline, PsycINFO, Scopus, CINAHL plus, Global Health and CAB Abstracts. These databases were searched due to their relevance and broad scope (including social and natural sciences, and medical literature). Grey literature was also searched using Open Grey (a multidisciplinary open access repository for grey literature in Europe which includes social science and biomedical science sources) (Public Health England Knowledge and Library Services, 2021). All databases were searched for the period 2000-2020 in June 2020 and limited to English. Searches of the databases were conducted by two research team members (RR and DS).

\subsection{Search terms}

A multidisciplinary team derived search terms for the three main concepts (population, exposure and outcome). Following previous research, we use a broad definition of anxiety and depression, including symptoms and precursors such as self-report of stress or low mood (Wolpert et al., 2019). In order to evaluate the role of exposure to green space in preventing anxiety and depression, ideally studies would include clinically measurable depression or anxiety as outcomes. However, we included a comprehensive list of terms to identify studies that may improve mental health or reduce symptoms of mental "disorder", as indicating reduction in risk of anxiety and depression. Terms including mental health, well-being, self-esteem and quality of life were included as these outcomes have been linked to anxiety and depression in the literature. As specific populations were not specified, these terms were expected to identify evidence about prevention of anxiety and depression in general populations. Similarly, exposure to green space/nature was broadly defined to include any length of time exposed to green space or nature (e.g. including one-off exposures and longer-term residential exposure). The search terms and strategy used for the Medline database are shown in Appendix 1. The same search terms were used across all databases, although the entry of the searches differed according to the requirements of each database.

\subsection{Study selection}

2.3.1 Eligibility criteria To be included in this scoping review, sources needed to have been published between 2000 and present, written in English and study human participants. There were no restrictions for source type, although experimental studies needed to include a comparator/control group. To provide a global scope, there was no restriction for country of publication. In terms of age of sample, eligibility was based on the age range of the 
sample, where given, or otherwise on the description of the sample (e.g. children, students, adults). Due to the lack of published literature focusing specifically on 14-24 age range, sources were included if the sample was between 14 and 24 years old, or at least some of the sample was within this age range. We decided to include sources with an overlapping age range to ensure we did not miss any studies including participants within the target age range. Sources were excluded if the outcome and/or exposure was out of scope, the population was not relevant for age or living in urban settings, the population's age was unknown, the full text could not be accessed or if only the abstract was available in English. Studies involving exposure to nature through technology were excluded, e.g. virtual reality.

2.3.2 Screening The search results were exported into Mendeley reference management software and duplicates removed, before title/abstract screening was carried out by one reviewer (IB). Full text screening was divided between four reviewers (IB, DS, RH, RR) with regular discussion about any cases that were unclear.

\subsection{Quality appraisal}

As the aim of this scoping review was to provide a broad, descriptive overview of the evidence (Munn et al., 2018), critical appraisal of the included studies was not carried out. Therefore, the quality of the studies was not determined.

\subsection{Data abstraction, charting and synthesis}

We planned to report where studies had been conducted, the study designs used, the nature of exposure and outcome, and characteristics of the sample. A data charting form was developed to record relevant data after full text screening. Data were extracted by one of four reviewers (IB, DS, RH, RR) on source characteristics; author, year of publication, title, journal, source type, country of origin, study type, population demographics, whether the target population was general or experienced anxiety and/or depression (either self-reported or diagnosed), type of exposure to greenspace or intervention, the outcome measure, and a reason for exclusion if applicable.

\section{Results}

\subsection{Numbers and general characteristics of sources}

Searches identified 6,902 records from databases which, once deduplicated, resulted in 6,861 unique sources (Figure 1). There were 405 sources in total after title/abstract screening. After full text screening, there was a total of 201 sources included in the scoping review.

General characteristics of the sources were analysed to show frequencies and distribution of the sources (Table 1). Most sources were published in peer-reviewed journals (96\%). A majority of studies were either observational (e.g. cross-sectional surveys of exposure to residential green space) (34\%) or experimental (e.g. randomised controlled trials conducted in Asian countries with student populations exposed to forests) (28\%). A total of 32 of the sources included were qualitative (16\%) and 21 had a mixed methods design (10.5\%). Most studies were carried out in North America (28.2\%), followed by Europe (21.8\%) and Asia (15.8\%) (Figure 2). The UK was categorised separately from Europe and included 35 sources (17.3\%). Most studies were of the general population (94\%). The remainder of sources involved populations already clinically or self-diagnosed with anxiety and/or depression (6\%). 


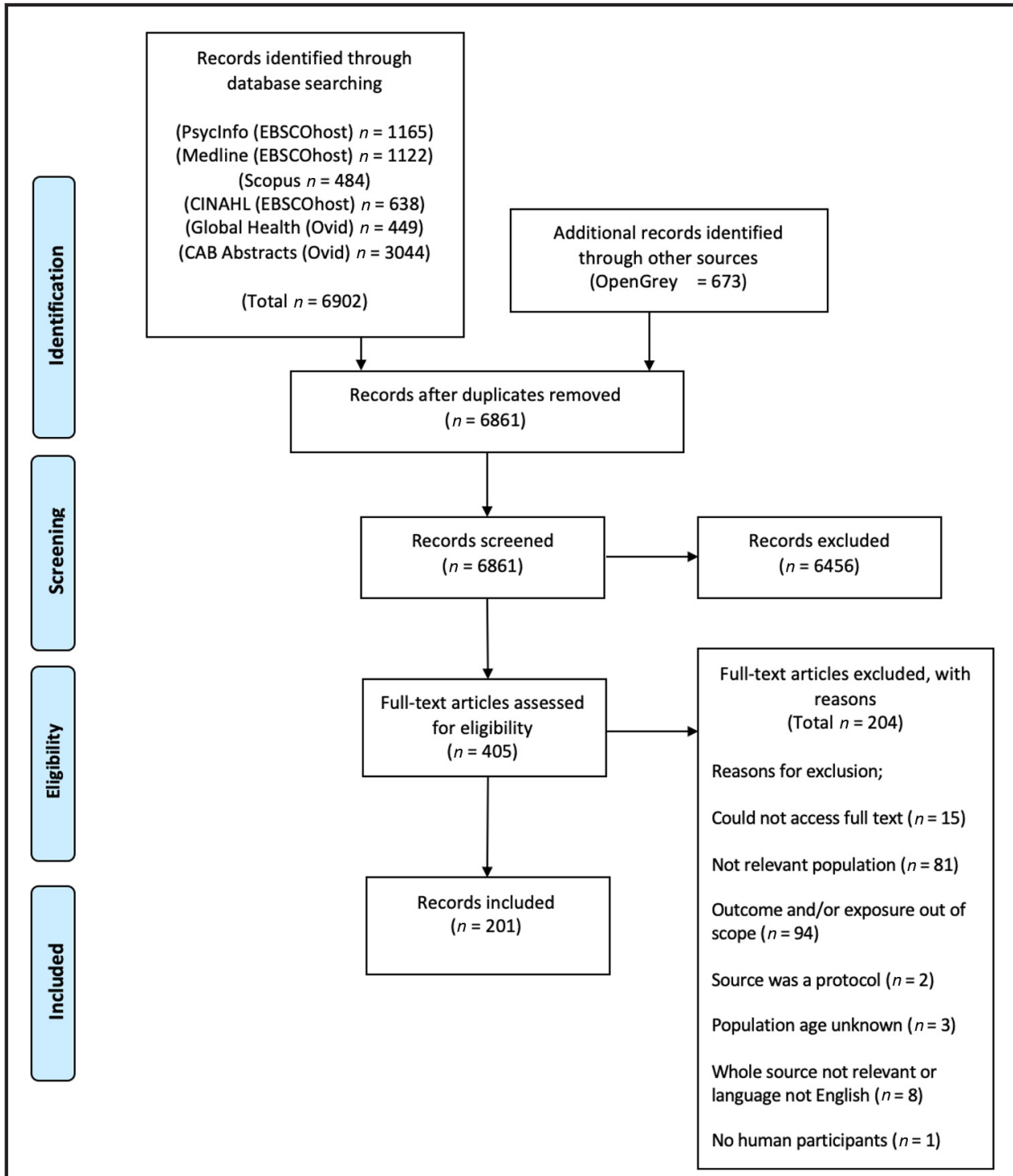

Note: Figure is based on Preferred Reporting Items for Systematic reviews and MetaAnalyses (PRISMA) guidelines

Source: Moher et al. (2009)

\subsection{Timing of publications}

Figure 3 shows the steady increase over time from 2000 to 2019 in the number of publications addressing exposure to green space and mental health of young people globally, which is particularly notable since 2012 .

\subsection{Exposure/intervention}

Sources were categorised into one of 17 categories based on the reported type of exposure or intervention. Urban green space (e.g. parks) was the most prevalent exposure type in the literature. Having contact with nature (e.g. touching natural 
Table 1 Characteristics of included sources

Characteristic

Number (\%)

Source type

Published article in peer-reviewed journal

$193(96)$

Doctoral thesis

$6(3)$

Report or book

Study design

Experimental

$57(28)$

Observational

68 (34)

Qualitative

$32(16)$

Mixed Methods

$21(10.5)$

Case Study

$4(2)$

Review (systematic or narrative)

$17(8.5)$

Other

Outcome Category

Anxiety

$17(8.5)$

Depression

$11(5.4)$

Both

$26(13)$

Other (mood, well-being, stress, quality of life, self-esteem)

$147(73.1)$

Age

Child OR an age range that is younger than 14-24 but overlaps with it OR adolescents

$70(35)$

$44(22)$

14-24 years OR within this age band OR students OR young people

$55(27)$

Children AND adults

$32(16)$

Ethnicity

Unspecified

$198(98.5)$

African American

$2(1)$

Latino

Target population

General

$188(94)$

Anxiety AND/OR depression

$13(6)$

Exposure/Intervention

Urban green space

$41(20)$

Exposure/contact/touching materials from nature

$40(19.5)$

Forest/woodland

$25(12)$

Camp/day trip

Other outdoor physical activity (e.g. rock climbing)

$21(10.5)$

$18(9)$

Outdoor walking

$16(7.5)$

Adventure therapy

$8(3.5)$

$5(2.5)$

$5(2.5)$

Other

4 (2)

$\begin{array}{ll}\text { Community gardens } & 4(2) \\ \text { Urban agriculture } & 4(2) \\ \text { Connection with nature } & 4(2)\end{array}$

$\begin{array}{lr}\text { Community gardens } & 4(2) \\ \text { Urban agriculture } & 4(2) \\ \text { Connection with nature } & 4(2)\end{array}$

Connection with nature $4(2)$

Blue spaces

$4(2)$

Outdoor running

$3(1.5)$

$2(1)$

Private gardens

$1(0.5)$

Note: $N=201$

materials) was also a common exposure type, after urban green space. Exposure to forest or woodland settings also dominated the literature and the majority of these studies were conducted in Asian countries. Exposure to green spaces through camps and adventure therapy was often reported from studies in the UK and North America. 


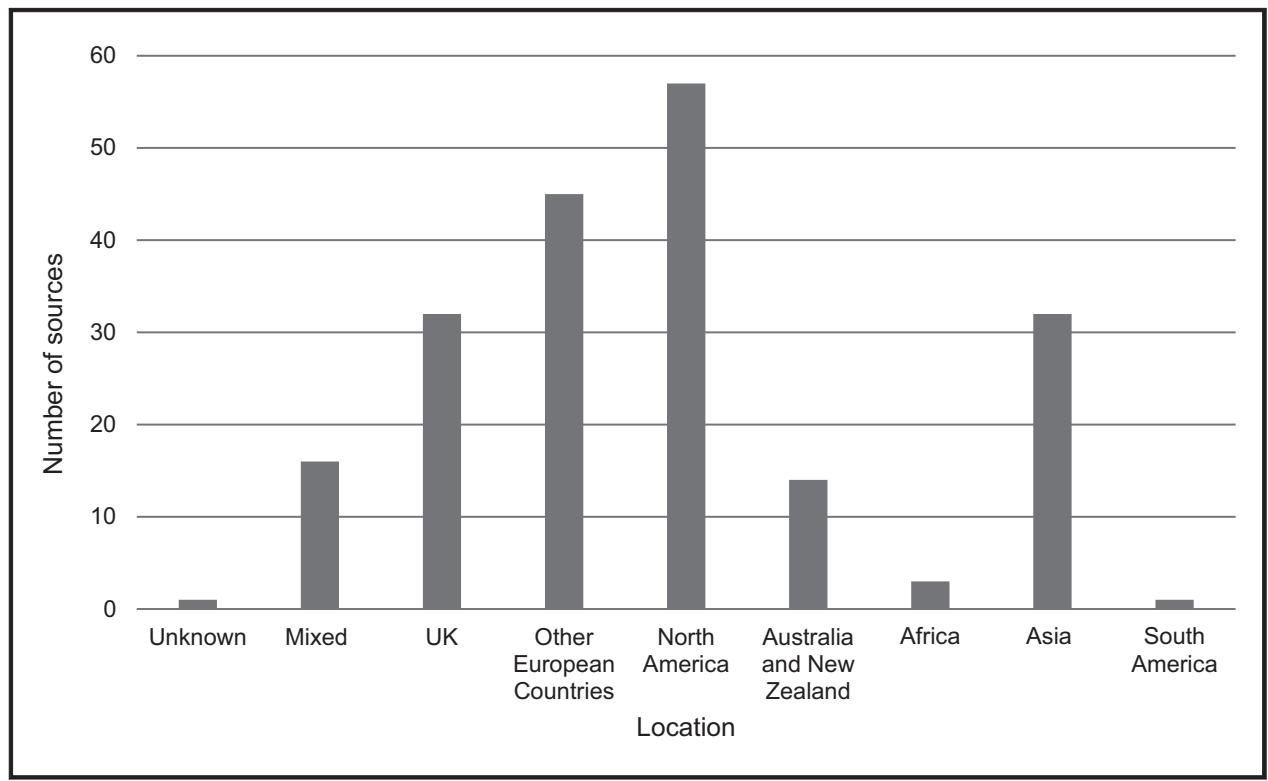

\section{Figure 3 Number of publications from 2000 to 2019}

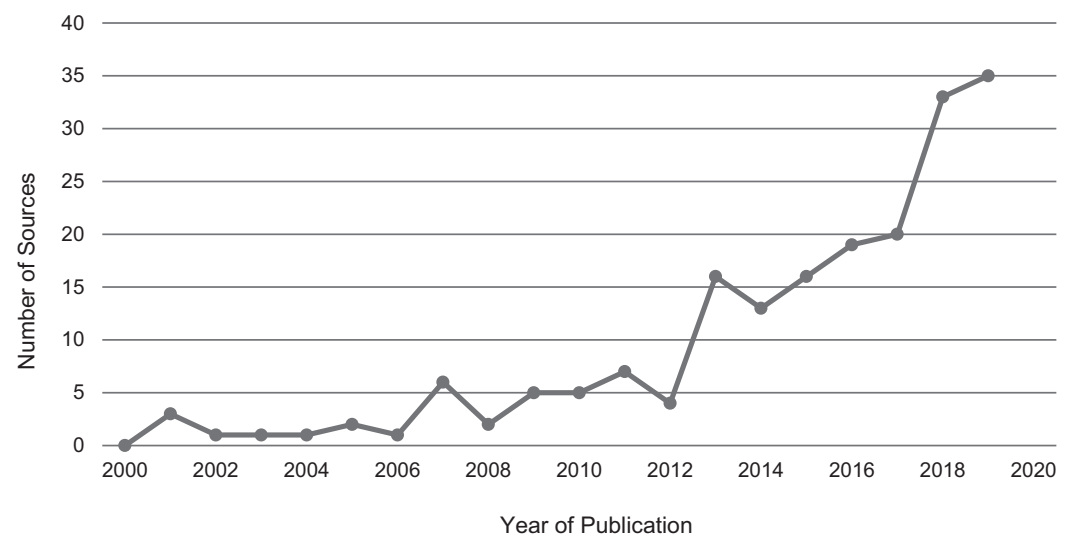

Another prevalent exposure to green spaces was through doing outdoor physical activity, including walking and running, and these sources had mainly experimental study designs. Studies involving exposure to blue spaces and outdoor employment were some of the least commonly reported, however notably these sources were published more recently. There were also fewer studies which reported exposure to green spaces through farms, private or community gardens, urban agriculture (e.g. allotments), and private gardens.

\subsection{Study outcomes}

Outcomes of interest were anxiety and depression, and any level and definition of these terms was included in our search strategy, e.g. clinical measures or self-reported 
symptoms or precursors of anxiety and depression, such as low mood. We found that few studies used clinical measures of anxiety or depression. Instead, for example, many studies measured anxiety using the Profile of Mood States scale (McNair and Lorr, 1964).

Therefore, the outcomes reported for most studies were defined as "Other", which included well-being, stress, quality of life, mood, mental health and physiological measures.

\section{Discussion}

\subsection{Main findings of this study}

The research question for this global scoping review was, "What is the state of the evidence base for exposure to green space to reduce the risk of anxiety and depression among young people living in urban settings?". The review highlighted that relevant studies have used a variety of study designs. The results showed that research in the field is global and publications are increasing. Further, the results showed a wide range of exposures to green spaces were studied, most studies reported more general mental health outcomes rather than clinical measures of anxiety or depression, and that most studies were conducted in North America or Europe.

\subsection{What is already known on this topic}

There have been several systematic reviews conducted showing exposure to green space and connection to nature as a way to benefit mental well-being for children and adults (McCormick, 2017; Vanaken and Danckaerts, 2018; Tillmann et al., 2018; Corazon et al., 2019; Houlden et al., 2018; Thompson Coon et al., 2011). The majority of the studies included in these reviews reported positive associations between green spaces and mental well-being. Further, there have been two recent scoping reviews describing the literature on the mental health benefits of green spaces (Wendelboe-Nelson et al., 2019; Callaghan et al., 2020). These scoping reviews did not focus on young people and included studies of treatment rather than prevention of mental health problems.

\subsection{What this study adds}

This scoping review has focused on young people specifically and described the state of the evidence regarding exposure to green spaces and reducing the risk of anxiety and depression. This adds to the literature because there is a lack of focus on young people in this area. This scoping review informs future research by describing the available literature for a systematic review, and highlighting gaps in the literature. For example, it shows that there are a limited number of studies with a sample within the age range 14-24years, and that most studies are carried out in high-income countries. Future studies should include clinical measures of anxiety and depression and should be carried out in a wider range of geographical and ethnic populations.

\subsection{Limitations of the literature}

This global scoping review showed that the published literature in the field was predominantly from high-income countries and that there was an under-representation of low-middle income countries (LMIC). It is important to know whether exposure to green spaces is beneficial in these countries, because there is a lack of mental health services in LMICs (Lund, 2020), and this could be a cost-effective preventive measure, particularly in high density cities. It has also highlighted the under-representation of different ethnicities in the literature. Furthermore, most outcomes reported in the literature were categorised as "Other" (not anxiety or depression). These outcomes included well-being, stress, quality of life, low mood and general mental 
health/illness/disorder/condition. There is limited literature using clinical measures of anxiety or depression as the primary outcome. Rather, broader terms for mental health which can be related to, or precursors to, anxiety or depression were more common in the literature.

\section{Conclusion}

In sum, this global scoping review has shown that the types of exposures to green space reported in the studies reviewed are very diverse. There is a lack of literature using clinical measures of anxiety and depression as a primary outcome, and many studies report other related outcomes (e.g. well-being, quality of life, stress). The research in the field is increasing, although this review has highlighted that the majority of studies are conducted in high-income countries. More research is needed from low- and middle-income countries, especially as these countries tend to have a less developed mental health services. Nevertheless, the findings presented here can be used to inform the focus of future systematic reviews on the prevention of anxiety and depression in young people aged 14-24years by describing the scope of the literature in terms of year of publication, geographic location and other characteristics of the sample, as well as a wide range of exposures. The heterogeneity of outcomes and exposures reported here could make this challenging.

\section{References}

Arksey, H. and O'Malley, L. (2005), "Scoping studies: towards a methodological framework", International Journal of Social Research Methodology, Vol. 8 No. 1, pp. 19-32.

Callaghan, A., McCombe, G., Harrold, A., McMeel, C., Mills, G., Moore-Cherry, N. and Cullen, W. (2020), "The impact of green spaces on mental health in urban settings: a scoping review", Journal of Mental Health, pp. 1-15.

Cao, W., Fang, Z., Hou, G., Han, M., Xu, X., Dong, J. and Zheng, J. (2020), "The psychological impact of the COVID-19 epidemic on college students in China", Psychiatry Research, Vol. 287, p. 112934.

Corazon, S.S., Sidenius, U., Poulsen, D.V., Gramkow, M.C. and Stigsdotter, U.K. (2019), "Psycho-physiological stress recovery in outdoor nature-based interventions: a systematic review of the past eight years of research", International Journal of Environmental Research and Public Health, Vol. 16 No. 10, p. 1711.

Houlden, V., Weich, S., de Albuquerque, J.P., Jarvis, S. and Rees, K. (2018), "The relationship between greenspace and the mental wellbeing of adults: a systematic review", PLoS One, Vol. 13 No. 9, pp. 1-35.

Jacka, F.N. and Reavley, N.J. (2014), "Prevention of mental disorders: evidence, challenges and opportunities", BMC Medicine, Vol. 12 No. 1.

Kessler, R.C., Amminger, G.P., Aguilar-Gaxiola, S., Alonso, J. and Lee, S. (2007), "Age of onset of mental disorders: a review of recent literature”, Current Opinion in Psychiatry, Vol. 20 No. 4, pp. 359-364.

Krefis, A., Augustin, M., Schlunzen, K., Ossenbrugge, J. and Augustin, J. (2018), "How does the urban environment affect health and well-being? A systematic review", Urban Science, Vol. 2 No. 1, pp. 21.

Lecic-Tosevski, D. (2019), "Is urban living good for mental health?", Current Opinion in Psychiatry, Vol. 32 No. 3, pp. 204-209.

Lund, C. (2020), "Reflections on the next ten years of research, policy and implementation in global mental health", Epidemiology and Psychiatric Sciences, Vol. 29, pp. 1-3.

McCormick, R. (2017), "Does access to green space impact mental well-being of children: a systematic review", Journal of Pediatric Nursing, Vol. 37, pp. 3-7.

McNair, D.M. and Lorr, M. (1964), "An analysis of mood in neurotics", The Journal of Abnormal and Social Psychology, Vol. 69 No. 6, pp. 620-627.

Mays, N., Roberts, E. and Popay, J. (2001), "Synthesizing research evidence", in Fulop, N., Allen, P., Clarke, A., and Black, N. (Eds), Studying the Organisation and Delivery of Health Services: Research Methods, Routledge, London, pp. 188-219.

Moher, D., Liberati, A., Tetzlaff, J. and Altman, D.G. (2009), "Preferred reporting items for systematic reviews and Meta-analyses: the PRISMA statement”, BMJ, Vol. 339 No. 1, p. b2535. 
Munn, Z., Peters, M., Stern, C., Tufanaru, C., McArthur, A. and Aromataris, E. (2018), "Systematic review or scoping review? Guidance for authors when choosing between a systematic or scoping review approach", BMC Medical Research Methodology, Vol. 18 No. 1.

Office for National Statistics (ONS) (2020), "Young people's well-being in the UK: 2020", [online], available at: www.ons.gov.uk/peoplepopulationandcommunity/wellbeing/bulletins/youngpeopleswellbeingintheuk/2020, (accessed 8 March 2021).

Patel, V., Flisher, A.J., Hetrick, S. and McGorry, P. (2007), "Mental health of young people: a global public-health challenge", The Lancet, Vol. 369 No. 9569, pp. 1302-1313.

Public Health England Knowledge and Library Services (2021), "Index of grey literature and alternative sources and resources", https://phelibrary.koha-ptfs.co.uk/greylit/ (accessed 25 February 2021).

Slater, S.J., Christiana, R.W. and Gustat, J. (2020), "Recommendations for keeping parks and green space accessible for mental and physical health during COVID-19 and other pandemics", Preventing Chronic Disease, Vol. 17, p. E59.

Srivastava, K. (2009), "Urbanization and mental health”, Industrial Psychiatry Journal, Vol. 18 No. 2, pp. 75-76.

Sundquist, K., Frank, G. and Sundquist, J. (2018), "Urbanisation and incidence of psychosis and depression", British Journal of Psychiatry, Vol. 184 No. 4, pp. 293-298.

Thomas, E., Serwicka, I. and Swinney, P. (2015), "Urban demographics. Why people live where they do. Center for cities", 10.13140/RG.2.1.1053.8965 (accessed 8 March 2021).

Thompson Coon, J., Boddy, K., Stein, K., Whear, R., Barton, J. and Depledge, M.H. (2011), "Does participating in physical activity in outdoor natural environments have a greater effect on physical and mental wellbeing than physical activity indoors? A systematic review", Environmental Science \& Technology, Vol. 45 No. 5, pp. 1761-1772.

Tiller, J. (2013), "Depression and anxiety”, Medical Journal of Australia, Vol. 199 No. 6, pp. 28-31.

Tillmann, S., Tobin, D., Avison, W. and Gililand, J. (2018), "Mental health benefits of interactions with nature in children and teenagers: a systematic review", Journal of Epidemiology and Community Health, Vol. 72 No. 10, pp. 958-966.

Tricco, A.C., Lillie, E., Zarin, W., O’Brien, K.K., Colquhoun, H., Levac, D., Moher, D., Peters, M.D., Horsley, T., Weeks, L. and Hempel, S. (2018), "PRISMA extension for scoping reviews (PRISMA-ScR): checklist and explanation", Annals of Internal Medicine, Vol. 169 No. 7, pp. 467-473.

UN Department of Economic and Social Affairs (2018), "68\% Of the world population projected to live in urban areas by 2050, says UN", [online], available at: www.un.org/development/desa/en/ news/population/2018-revision-of-world-urbanization-prospects.html, (accessed 12 March 2020).

Vanaken, G. and Danckaerts, M. (2018), "Impact of green space exposure on children's and adolescents' mental health: a systematic review", International Journal of Environmental Research and Public Health, Vol. 15 No. 12, pp. 12.

Ventriglio, A., Torales, J., Castaldelli-Maia, J., De Berardis, D. and Bhugra, D. (2021), "Urbanization and emerging mental health issues", CNS Spectrums, Vol. 26 No. 1, pp. 43-50.

Wendelboe-Nelson, C., Kelly, S., Kennedy, M. and Cherrie, J.W. (2019), "A scoping review mapping research on green space and associated mental health benefits", International Journal of Environmental Research and Public Health, Vol. 16 No. 12, p. 2081.

Wolpert, M., Dalzell, K., Ullman, R., Garland, L., Cortina, M., Hayes, D., Patalay, P. and Law, D. (2019), "Strategies not accompanied by a mental health professional to address anxiety and depression in children and young people: a scoping review of range and a systematic review of effectiveness", The Lancet Psychiatry, Vol. 6 No. 1, pp. 46-60.

World Health Organisation, WHO (2017), "Depression and other common mental disorders: global health estimates. [online]", available at: https://apps.who.int/iris/bitstream/handle/10665/254610/WHO-MSDMER-2017.2-eng.pdf, (accessed 11 March 2021).

World Health Organisation, WHO (2020), "Mental health", [online] available at: www.who.int/healthtopics/mental-health\#tab=tab_2, (accessed 3 August 2020).

Worldometer (2020), "Population by gender, age, fertility rate, immigration", [online], available at: www. worldometers.info/worldpopulation/world-population-gender-age.php, (accessed 12 March 2020). 


\section{Appendix}

\section{Table A1 Search terms used in Medline}

Concept

Population (searched in abstract)

Exposure (searched in title only)

Outcome (searched in abstract)

NOT (searched in full text)

\section{Synonym}

Young OR adolescen* OR adult OR teen* OR youth OR juvenile OR male OR female OR child*

Natur* OR green* OR blue OR open-space* OR "open space*" OR park OR field* OR lake OR wood* OR forest OR garden OR sea OR coast OR water OR allotment OR mountain OR hill* OR farm OR "protected area" OR reserve OR tree OR habitat OR biodivers* OR outdoor* OR wildlife OR river OR canal OR farm OR trail OR mountain* OR trek* OR walk* OR hike OR hiking OR camp* OR canoe* OR row* OR climb* OR expedition OR run* OR ski* OR ride OR bike OR cycl* OR tour OR hash OR pony OR horse OR conserv* OR (environment* AND volunt*)

Anxiety OR depression OR stress OR "low mood" OR depressed OR anxious OR psychological OR mental OR illness OR disorder OR condition OR phobia OR agoraphobia OR wellbeing OR wellbeing schizophrenia OR PTSD OR spider OR psychosis OR pain OR cancer OR survivor OR epilepsy OR IBS OR IBD OR disease OR maternal OR menstrual OR eating OR TRAILS OR exp animals/ not humans.sh

\section{Corresponding author}

Rebecca Reece can be contacted at: rebecca.reece@uwe.ac.uk

For instructions on how to order reprints of this article, please visit our website: www.emeraldgrouppublishing.com/licensing/reprints.htm

Or contact us for further details: permissions@emeraldinsight.com 